\title{
Quantum-state preparation and control via the Zeno effect
}

\author{
Alfredo Luis* \\ Departamento de Óptica, Facultad de Ciencias Físicas, Universidad Complutense, 28040 Madrid, Spain
}

(Received 12 November 2000; published 18 April 2001)

\begin{abstract}
We present different schemes for preparing and maintaining the quantum state of multilevel systems. This is achieved via a suitable combination of coherent trapping and the Zeno effect which serves to protect the prepared state against undesired jumps to other states.
\end{abstract}

DOI: $10.1103 /$ PhysRevA.63.052112

PACS number(s): 03.65.Xp, 42.50.- p, 32.80.-t

\section{INTRODUCTION}

In recent times there has been increasing interest in the engineering of quantum states [1]. This interest has been prompted by the continual progress in the practical application of quantum theory, which usually demands the tailoring of specific states (usually highly nonclassical) in order to develop the possibilities offered by the quantum framework (for example, to encode and process quantum information, or to perform the most accurate measurements).

In this work we propose practical schemes for preparing the quantum state of multilevel systems. The objective is that such preparation must be robust, i.e., the prepared state must endure couplings and interactions that otherwise would alter it. We will demonstrate that this can be achieved via a suitable combination of coherent trapping, adiabatic following, and the Zeno effect. Compared to other proposals $[2,3]$, the schemes introduced in this work are simpler, more versatile, and can be applied to systems with an arbitrary number of levels.

The Zeno effect refers to the inhibition of the isolated dynamics of a system when the evolution is observed with enough resolution [4]. In our context this can be used to protect or block a given state by preventing undesired transitions to other states [5,6]. Moreover, the Zeno effect allows us to replace the original dynamics by a prescribed evolution via suitably designed time-dependent measurements (inverse Zeno effect) $[2,7]$. Therefore, the concept of the Zeno effect serves to tailor the time evolution of an observed system.

Perhaps the most important field of potential applications of this idea would be the control on demand of the internal electronic states of atoms and their time evolution. We can include the monitoring of the vibrational states of trapped ions and molecules, for example. These applications are very interesting because the interaction between matter and light, and its use in manipulating one by the other, is of fundamental importance in quantum optics.

In this work we propose three different schemes for preparing and controlling the quantum state of multilevel systems. They are introduced in Sec. II, focusing on the simplest nontrivial example of a two-level system. In Sec. III we generalize them to the case of an arbitrary number of levels.

*Electronic address: alluis@eucmax.sim.ucm.es

\section{STATE PREPARATION AND CONTROL IN TWO-LEVEL SYSTEMS}

In this section we present three different procedures for preparing a two-level system in a given robust state. For simplicity we find it helpful to refer to two internal atomic levels (i.e., a two-level atom), but the procedures can be applied to other practical situations.

All the proposals will be based on the three-level arrangement with $\Lambda$ configuration schematized in Fig. 1. The system of interest is spanned by the metastable vectors $|1\rangle,|2\rangle$. These two levels are coupled via two coherent laser fields to an auxiliary level $|r\rangle$. The laser fields can be detuned and we assume that the detuning $\Delta$ is the same for both. The level $|r\rangle$ can be unstable, decaying spontaneously to another auxiliary level $|\ell\rangle$ at rate $\gamma$. Different schemes are obtained depending on the particular values of $\gamma$ and $\Delta$. For simplicity we assume that $|r\rangle$ does not decay to $|1\rangle$ and $|2\rangle$. Nevertheless, below we will show that the same final results are obtained even if $|r\rangle$ does decay to $|1\rangle$ and $|2\rangle$.

\section{A. Resonant coupling to an unstable level}

In this first proposal the driving fields are resonant $(\Delta$ $=0)$ and the auxiliary state $|r\rangle$ is unstable $(\gamma \neq 0)$. The dynamics of the whole system is described by the following master equation, in the interaction picture and units where $\hbar=1$, for the density matrix $\rho$ :

$$
\dot{\rho}=-i\left[H_{\Omega}, \rho\right]-\frac{\gamma}{2}(|r\rangle\langle r|\rho+\rho| r\rangle\langle r|-2| \ell\rangle\langle r|\rho| r\rangle\langle\ell|)
$$

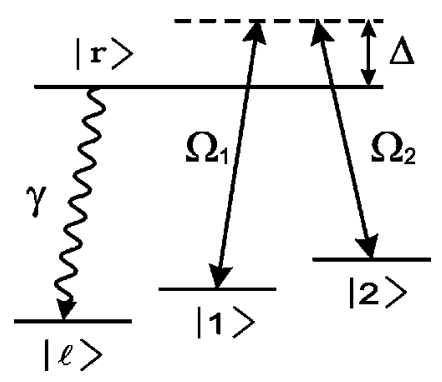

FIG. 1. Level diagram for preparing robust linear combinations of the metastable states $|1\rangle$ and $|2\rangle$. These levels are coupled by two laser fields to an auxiliary level $|r\rangle$ that can decay to the level $|\ell\rangle$ at rate $\gamma$. The parameters $\Omega_{1}$ and $\Omega_{2}$ denote the strength of the laser couplings and $\Delta$ is the detuning. The three cases discussed in the text are $(A) \quad \gamma \neq 0, \Delta=0 ;(B) \quad \gamma=0, \Delta=0 ;(C) \quad \gamma=0, \Delta$ $\neq 0$. 


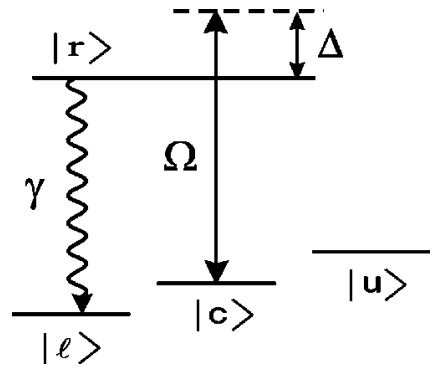

FIG. 2. Level scheme fully equivalent to Fig. 1 in terms of the coupled $|c\rangle$ and uncoupled $|u\rangle$ states.

with

$$
H_{\Omega}=\sum_{k=1}^{2}\left(\Omega_{k}^{*}|r\rangle\left\langle k\left|+\Omega_{k}\right| k\right\rangle\langle r|\right),
$$

where $\Omega_{k}$ are constants depending on the phase and intensity of the driving beams.

The analysis of the dynamics can be simplified if we use the basis

$$
\begin{aligned}
& |c\rangle=\frac{1}{\Omega}\left(\Omega_{1}|1\rangle+\Omega_{2}|2\rangle\right), \\
& |u\rangle=\frac{1}{\Omega}\left(\Omega_{2}|1\rangle-\Omega_{1}|2\rangle\right),
\end{aligned}
$$

where $\Omega=\sqrt{\Omega_{1}^{2}+\Omega_{2}^{2}}$. In this basis the Hamiltonian is

$$
H_{\Omega}=\Omega(|r\rangle\langle c|+| c\rangle\langle r|),
$$

and the level scheme in Fig. 1 can be replaced by the simpler one in Fig. 2 with $\Delta=0$. We can see that the only state coupled to the auxiliary level $|r\rangle$ is $|c\rangle$ (coupled state). Due to the unstable character of $|r\rangle$ all the population in $|c\rangle$ will be irreversibly transferred to $|\ell\rangle$ and removed from the system.

On the other hand, the state $|u\rangle$ is completely decoupled from the rest of the levels. Since $H_{\Omega}|u\rangle=0$ this state is stationary (within the interaction picture). If the atom is prepared in $|u\rangle$ it will remain always in the same state. In other words, this procedure is a coherent trapping and $|u\rangle$ is the trapped or dark state [8].

Any linear combination of $|1\rangle$ and $|2\rangle$ can be prepared in this way simply by choosing the appropriate coupling constants $\Omega_{1}$ and $\Omega_{2}$. Moreover, it is possible to change the prepared state at will simply by suitably varying $\Omega_{1}$ and $\Omega_{2}$. The only requisite is that such a variation must be adiabatic: $\Omega_{1}(t), \Omega_{2}(t)$ must vary slowly so that at any time $H_{\Omega}(t)|u(t)\rangle=0$ (adiabatic following) [8].

The prepared state $|u\rangle$ depends only on the relative value $\Omega_{1} / \Omega_{2}$ of the coupling strengths. The absolute value $\Omega$ is a free parameter that can be used to render the state $|u\rangle$ stable and robust. More specifically, the purpose is to prevent coherent transitions from $|u\rangle$ to $|c\rangle$. This can be achieved via the Zeno effect since the transition $|c\rangle \rightarrow|r\rangle$ and the spontaneous emission of a photon when $|r\rangle$ decays to $|\ell\rangle$ can be regarded as a continuous measurement of whether the transition from $|u\rangle$ to $|c\rangle$ has occurred or not. The emission of the photon is evidence that the system was in the state $|c\rangle$ instead of $|u\rangle$. For suitable $\Omega$ and $\gamma$ the measurement can be accurate enough so that the Zeno effect occurs: i.e., the evolution from $|u\rangle$ to $|c\rangle$ is prevented and the system is blocked in the desired state $|u\rangle[9]$.

To examine this possibility in quantitative terms we include in the dynamics an interaction term $H_{\omega}$ inducing $|u\rangle$ $\rightarrow|c\rangle$ transitions,

$$
H_{\omega}=\omega(|u\rangle\langle c|+| c\rangle\langle u|) .
$$

The evolution of the system is given by Eq. (2.1), replacing $H_{\Omega}$ by $H_{\Omega}+H_{\omega}$. It can be easily seen that the evolution equations for all the matrix elements involving only the states $|c\rangle,|u\rangle$, and $|r\rangle$ form a closed set. This allows us to simplify the problem since the unnormalized projection of the whole system into the subspace spanned by $|c\rangle,|u\rangle$, and $|r\rangle$ (we will denote such a projection as $|\psi\rangle$ ) evolves according to the following Schrödinger equation with complex Hamiltonian:

$$
|\dot{\psi}\rangle=-i\left(H_{\Omega}+H_{\omega}-i \frac{\gamma}{2}|r\rangle\langle r|\right)|\psi\rangle .
$$

The projection $|\psi\rangle$ is a pure state because the initial state $|u\rangle$ is pure. If we express $|\psi\rangle$ as

$$
|\psi\rangle=\alpha_{c}|c\rangle+\alpha_{u}|u\rangle+\alpha_{r}|r\rangle,
$$

we get the following equations of motion for the coefficients:

$$
\begin{gathered}
\dot{\alpha}_{c}=-i \omega \alpha_{u}-i \Omega \alpha_{r}, \\
\dot{\alpha}_{u}=-i \omega \alpha_{c}, \\
\dot{\alpha}_{r}=-i \Omega \alpha_{c}-\frac{\gamma}{2} \alpha_{r} .
\end{gathered}
$$

Our initial conditions are $\alpha_{c}=\alpha_{r}=0$ and $\alpha_{u}=1$.

These equations can be easily solved. However, we can simplify them further since we must consider system parameters leading to a good observation regime of the moment in which the $|u\rangle \rightarrow|c\rangle$ transition occurs. This means that the following relations should be satisfied [9]:

$$
\gamma, \Omega, \frac{\Omega^{2}}{\gamma} \gg \omega
$$

This implies that as soon as the system is in the state $|c\rangle$ a $|c\rangle \rightarrow|r\rangle$ transition takes place and $|r\rangle$ immediately decays to $|\emptyset\rangle$ with the emission of a photon. This takes place in a time interval short when compared to the period of the $|c\rangle \leftrightarrow|u\rangle$ oscillation caused by $H_{\omega}$.

The conditions (2.9) introduce two different time scales to the problem that allow us to eliminate rapid transients, which are invisible in the coarse-grained time scale of interest. A good approximation of Eqs. (2.8) is obtained by considering that $\alpha_{r}$ is always in its steady-state value, $\alpha_{r}=$ 
$-(2 i \Omega / \gamma) \alpha_{c}$, which adiabatically follows the evolution of $\alpha_{c}$. This reduces Eqs. (2.8) to

$$
\begin{gathered}
\dot{\alpha}_{c}=-\frac{2 \Omega^{2}}{\gamma} \alpha_{c}-i \omega \alpha_{u}, \\
\dot{\alpha}_{u}=-i \omega \alpha_{c} .
\end{gathered}
$$

Since $\Omega^{2} \gg \omega \gamma$ we can simplify Eqs. (2.10) by considering that $\alpha_{c}$ is always in its steady-state value $\alpha_{c}$ $=-i \gamma \omega \alpha_{u} /\left(2 \Omega^{2}\right)$. This leads to

$$
\dot{\alpha}_{u}=-\frac{\gamma \omega^{2}}{2 \Omega^{2}} \alpha_{u},
$$

and finally

$$
\alpha_{u}(t)=e^{-\left(\gamma \omega^{2} / 2 \Omega^{2}\right) t}
$$

The conclusion is that it is possible to protect the state $|u\rangle$ against disturbing couplings during a time interval $\tau$ given by

$$
\tau \ll \frac{2 \Omega^{2}}{\gamma \omega^{2}} .
$$

If $t \leqslant \tau$ we have that $|\psi(t)\rangle \simeq|\psi(0)\rangle=|u\rangle$. This time interval can be made arbitrarily large simply by increasing $\Omega$.

Because of conditions (2.9) this time interval encompasses many periods of the potential oscillation caused by $H_{\omega}$. Therefore, this preservation of the initial state $|u\rangle$ is an example of the coherent Zeno effect in which the observation prevents a coherent transition $|u\rangle \rightarrow|c\rangle$. On the other hand, this result can also be regarded as an example of the incoherent Zeno effect, which takes place when an irreversible decay is halted. This is because the initial state $|u\rangle$ is unstable because of the cascade of transitions

$$
|u\rangle \rightarrow|c\rangle \rightarrow|r\rangle \rightarrow|\ell\rangle .
$$

This is an example of so-called reservoir engineering where a metastable state becomes unstable on demand [10]. The interpretation as an incoherent Zeno effect has the peculiarity that the observing apparatus is involved in the mechanism causing the decay.

To conclude this point, this same scheme can be regarded also as a potential implementation of the so-called inverse Zeno effect: i.e., dynamics caused and controlled by measurement $[2,7]$. This occurs if $\Omega_{1}$ and $\Omega_{2}$ are varied adiabatically. In such a case the measuring arrangement is detecting the population of the time-dependent states $|c(t)\rangle$ and $|u(t)\rangle$. In the appropriate limit, the measurement forces the system to be always in the state $|u(t)\rangle$ evolving as dictated by the measuring arrangement, irrespective of any other dynamical influences.

We have shown that if the atom is prepared in the state $|u\rangle$ it will always remain in the same state. Next we address the initial preparation. In the scheme just analyzed we have that after a suitable time interval all the population of the coupled state $|c\rangle$ will be completely removed from the system of interest due to the spontaneous decay of the level $|r\rangle$. Thus, when the stationary state is reached all the atomic population in levels $|1\rangle$ and $|2\rangle$ is necessarily in the dark state $|u\rangle$ irrespective of the initial conditions.

We can briefly discuss another procedure for preparing the initial state of the system which is also valid for more general schemes, including the examples to be analyzed below. Using the above mentioned adiabatic transfer it is possible to prepare the two-level system in the desired state starting from any other state (say $|u\rangle=|1\rangle$, for instance) and no fields $\left(\Omega_{1}=\Omega_{2}=0\right)$ as the initial conditions. In the first place the field $\Omega_{2}$ should be turned on while $\Omega_{1}=0$. This step does not require any special care since the transition from 0 to $\Omega_{2}$ does not perturb the decoupled atomic state $|u\rangle=|1\rangle$ in any case. Then the other field $\Omega_{1}$ can be turned on. In this case the transition from 0 to $\Omega_{1}$ must be done slowly enough so that the dark state evolves adiabatically. Otherwise, a sudden turn on would significantly perturb the atomic state. This method can be applied provided that during these steps there are no external perturbations that might alter the atomic state before it comes to be protected by the Zeno effect.

Finally, we show that the same results are obtained after including the possibility of spontaneous transitions from $|r\rangle$ to $|1\rangle$ and $|2\rangle$. This is because once the Zeno effect freezes the system in the state $|u\rangle$ there are no further transitions to $|r\rangle$. Therefore it no longer matters how $|r\rangle$ would decay. The essential point is that the decay must be evidence that the atom leaves the state $|u\rangle$. Concerning the initial preparation of $|u\rangle$, the spontaneous decay of $|r\rangle$ to $|1\rangle$ and $|2\rangle$ would favor the population of $|u\rangle$.

\section{B. Resonant coupling to a metastable state}

Next we propose a slightly different example, also leading to the robust preparation of any desired state for a two-level system. The driving fields are again resonant $(\Delta=0)$, but in this case the level $|r\rangle$ is metastable $(\gamma \rightarrow 0)$ so that it does not decay to any other level during the time interval of interest. Nevertheless, we will see that the final result is the same obtained in the preceding subsection.

The levels $|c\rangle,|u\rangle$, and $|r\rangle$ form a closed system experiencing a purely unitary dynamics governed by the Hamiltonian $H_{\Omega}$ in Eq. (2.2). After the definitions (2.3) the interaction Hamiltonian becomes (2.4) and Fig. 1 becomes Fig. 2 with $\Delta=0$ and $\gamma=0$. Here again we have the same uncoupled state $|u\rangle$ which can be prepared in an arbitrary state by adiabatically varying $\Omega_{1}$ and $\Omega_{2}$.

To study the stability of $|u\rangle$ against couplings of the form (2.5) we have to solve the Schrödinger equation

$$
|\dot{\psi}\rangle=-i\left(H_{\Omega}+H_{\omega}\right)|\psi\rangle,
$$

with the initial condition $|\psi(0)\rangle=|u\rangle$. The solution leads to the following survival probability of the uncoupled state $|u\rangle$ : 


$$
P_{u}(t)=|\langle u \mid \psi(t)\rangle|^{2}=\frac{1}{\left(1+\eta^{2}\right)^{2}}\left[1+\eta^{2} \cos (\bar{\Omega} t)\right]^{2},
$$

where $\eta=\omega / \Omega$ and $\bar{\Omega}=\sqrt{\Omega^{2}+\omega^{2}}$.

We are interested in the conditions leading to the limit $P_{u}(t) \rightarrow 1$ for all times. This can be achieved provided that $\Omega \gg \omega$. In such a case $\eta \rightarrow 0$ and the survival probability can be approximated by

$$
P_{u}(t) \simeq 1-4\left(\frac{\omega}{\Omega}\right)^{2} \sin ^{2}(\bar{\Omega} t / 2),
$$

so that $P_{u}(t) \rightarrow 1$ when $\omega / \Omega \rightarrow 0$.

It can be asked whether this preservation of the uncoupled state can be regarded as a Zeno effect. It might be argued that no measurement is performed since $\gamma=0$ and the evolution is purely unitary. However, this evolution may be the initial stage of a measurement in which the observed system is unitarily coupled to the probe or meter. It has been shown that the Zeno effect can occur without completing the measurement provided that the probe carries relevant information about the system [11]. In our case the propagation of the field modes driving the $|c\rangle \leftrightarrow|r\rangle$ transition depends on whether the atomic state is $|c\rangle$ or $|u\rangle$, since they lead to a different polarization of the medium. Therefore, this blocking of the system in the state $|u\rangle$ may also be regarded as a Zeno effect. Nevertheless, a complete analysis of this point would require a quantum treatment of the driving fields. For further discussions about the relationship between the Zeno effect and coherent trapping with and without dissipation, see Ref. [12].

\section{Nonresonant coupling to a metastable state}

In this example the auxiliary level $|r\rangle$ is again metastable $(\gamma=0)$ but the driving fields are not resonant $(\Delta \neq 0)$ and we assume that the detuning $\Delta$ is the same for the two fields (two-photon resonance for the transition $|1\rangle \leftrightarrow|2\rangle$ ).

The space spanned by $|1\rangle,|2\rangle$, and $|r\rangle$ is a closed system and the dynamics, in a suitably defined interaction picture, is governed by the interaction Hamiltonian

$$
H_{\Omega}=-\Delta|r\rangle\langle r|+\sum_{k=1}^{2}\left(\Omega_{k}^{*}|r\rangle\left\langle k\left|+\Omega_{k}\right| k\right\rangle\langle r|\right)
$$

With the help of the definitions (2.3) this Hamiltonian can be written as

$$
H_{\Omega}=-\Delta|r\rangle\langle r|+\Omega(|r\rangle\langle c|+| c\rangle\langle r|) .
$$

Concerning the robustness of the dark state $|u\rangle$, we can show that it is stable against couplings such as (2.5) provided that the detuning $\Delta$ is large enough, $\Delta \gg \Omega$. In such a case no transitions $|r\rangle \leftrightarrow|c\rangle$ occur since the total Hamiltonian can be approximated by

$$
H_{\Omega}+H_{\omega} \simeq-(\Delta+\delta)|r\rangle\langle r|+\delta| c\rangle\langle c|+\omega(|u\rangle\langle c|+| c\rangle\langle u|),
$$

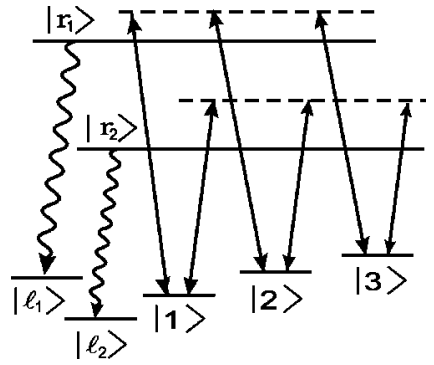

FIG. 3. Level system for preparing robust linear combinations of the metastable states $|1\rangle,|2\rangle$, and $|3\rangle$. Each level is coupled by two laser fields to two auxiliary levels $\left|r_{1}\right\rangle,\left|r_{2}\right\rangle$ that can decay to the levels $\left|\ell_{1}\right\rangle,\left|\ell_{2}\right\rangle$.

where $\delta=\Omega^{2} / \Delta$. We can see that $\delta$ acts as a detuning for the $|c\rangle \leftrightarrow|u\rangle$ transition. If $\delta \gg \omega$ we obtain an effective interaction Hamiltonian of the form

$$
H_{\Omega}+H_{\omega} \simeq-(\Delta+\delta)|r\rangle\langle r|-\epsilon| u\rangle\langle u|+(\delta+\epsilon)| c\rangle\langle c|,
$$

where $\epsilon=\omega^{2} / \delta$.

In these conditions we can conclude from Eq. (2.21) that $|u\rangle$ is a robust trapped state. Incidentally, we can also see in Eq. (2.21) that the states $|c\rangle$ and $|u\rangle$ play equivalent roles. Both are stationary (within the interaction picture) and both can be considered as robust trapped states.

\section{STATE PREPARATION AND CONTROL IN $N$-LEVEL SYSTEMS}

In this section we generalize the two-level schemes introduced in the preceding section to an arbitrary number of levels. In the first place we examine a three-level system because it properly illustrates the general case.

The case $N=3$ is illustrated in Fig. 3. The system of interest is spanned by the vectors $|1\rangle,|2\rangle$, and $|3\rangle$. Each level is coupled by coherent laser fields to two auxiliary levels $\left|r_{1}\right\rangle$ and $\left|r_{2}\right\rangle$. As in the preceding section we can consider three different cases: $(A)\left|r_{1}\right\rangle$ and $\left|r_{2}\right\rangle$ unstable and exact resonance; $(B)\left|r_{1}\right\rangle$ and $\left|r_{2}\right\rangle$ metastable and exact resonance; $(C)\left|r_{1}\right\rangle$ and $\left|r_{2}\right\rangle$ metastable and no resonance.

Here we will consider in some detail the case $B$. The cases $A$ and $C$ are straightforward once we have examined this one. In the interaction picture the Hamiltonian is

$$
H_{\Omega}=\sum_{j=1}^{2} \sum_{k=1}^{3}\left(\Omega_{k, j}^{*}\left|r_{j}\right\rangle\left\langle k\left|+\Omega_{k, j}\right| k\right\rangle\left\langle r_{j}\right|\right),
$$

where $\Omega_{k, j}$ are complex coupling constants that depend on the driving fields. In the most general case this Hamiltonian defines two coupled states $\left|c_{1}\right\rangle$ and $\left|c_{2}\right\rangle$,

$$
\left|c_{j}\right\rangle=\frac{1}{\Omega_{j}} \sum_{k=1}^{3} \Omega_{k, j}|k\rangle, \quad j=1,2,
$$

where $\Omega_{j}^{2}=\Sigma_{k=1}^{3}\left|\Omega_{k, j}\right|^{2}$. On the other hand, there is an uncoupled state defined by the orthogonality conditions $\left\langle c_{j} \mid u\right\rangle$ $=0$ for $j=1,2$. 


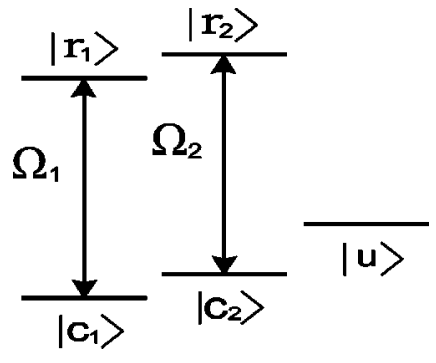

FIG. 4. Level scheme equivalent to Fig. 3 in terms of the coupled $\left(\left|c_{1}\right\rangle,\left|c_{2}\right\rangle\right)$ and uncoupled $(|u\rangle)$ states. In this case the levels $\left|r_{1}\right\rangle,\left|r_{2}\right\rangle$ do not decay and there is exact resonance.

In terms of these vectors the Hamiltonian can be written as

$$
H_{\Omega}=\sum_{j=1}^{2} \Omega_{j}\left(\left|r_{j}\right\rangle\left\langle c_{j}|+| c_{j}\right\rangle\left\langle r_{j}\right|\right)
$$

and the associated level diagram is schematized in Fig. 4. From Eq. (3.3) it is clear that $|u\rangle$ is a dark state $H_{\Omega}|u\rangle=0$. If the system is prepared in the state $|u\rangle$ it will always remain in the same state. Arbitrary linear combinations of $|1\rangle,|2\rangle$, and $|3\rangle$ can be prepared in this way simply by suitably varying the intensities and phases of the driving fields.

As before, the Zeno effect can be used to render the uncoupled state $|u\rangle$ stable against interactions of the form

$$
H_{\omega}=\sum_{j=1}^{2} \omega_{j}\left(|u\rangle\left\langle c_{j}|+| c_{j}\right\rangle\langle u|\right) \text {. }
$$

According to the results of the preceding section, the robustness can be achieved provided that $\Omega_{1}, \Omega_{2} \gg \omega_{1}, \omega_{2}$ since in such a case the transitions $|u\rangle \rightarrow\left|c_{j}\right\rangle$ are prevented.

Now we can easily generalize these results to an arbitrary number $N$ of levels. Formulas (3.1), (3.2), (3.3) and (3.4) are valid simply by extending the range of variation of the sums in the form $k=1,2, \ldots, N$ and $j=1,2, \ldots, N-1$. Each level is coupled to $N-1$ auxiliary levels $\left|r_{j}\right\rangle$. In the most general case this naturally defines $N-1$ coupled states $\left|c_{j}\right\rangle$ and one uncoupled state $|u\rangle$. The preparation of $|u\rangle$ is robust provided that the coupling constants $\Omega_{j}$ for the transitions $\left|c_{j}\right\rangle \leftrightarrow\left|r_{j}\right\rangle$ are strong enough to prevent $|u\rangle \rightarrow\left|c_{j}\right\rangle$ transitions.

Similar conclusions are obtained for the cases $A$ and $C$. Nevertheless, for scheme $C$ there is a much simpler generalization than the one just discussed. As we have mentioned above, for the case $C$ and $N=2$ the coupled and uncoupled states are equivalent. Taking advantage of this fact we can propose the generalization illustrated in Fig. 5, in which all levels are coupled to a single auxiliary state. When compared to the preceding one, this arrangement requires only one auxiliary level (instead of $N-1$ ) and only $N-1$ driving fields [instead of $N(N-1)$ ]. The Hamiltonian in the interaction picture is

$$
H_{\Omega}=-\Delta|r\rangle\langle r|+\sum_{k=1}^{N}\left(\Omega_{k}|k\rangle\left\langle r\left|+\Omega_{k}^{*}\right| r\right\rangle\langle k|\right)
$$

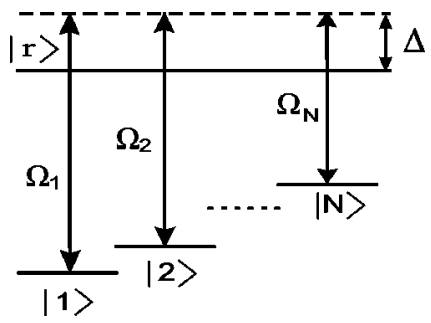

FIG. 5. Level diagram for preparing robust linear combinations of the $N$ levels $|1\rangle,|2\rangle, \ldots,|N\rangle$. Each level is coupled to the same auxiliary metastable level $|r\rangle$ by nonresonant laser fields. The parameters $\Omega_{j}, j=1,2, \ldots, N$, denote the strength of the couplings and $\Delta$ is the detuning.

which defines a single coupled state

$$
|c\rangle=\frac{1}{\Omega} \sum_{k=1}^{N} \Omega_{k}|k\rangle,
$$

where $\Omega^{2}=\sum_{k=1}^{N}\left|\Omega_{k}\right|^{2}$. The Hamiltonian can be written as

$$
H_{\Omega}=-\Delta|r\rangle\langle r|+\Omega(|c\rangle\langle r|+| r\rangle\langle c|),
$$

and the level scheme in Fig. 5 can be replaced by the simpler one in Fig. 6. Accordingly, there are $N-1$ uncoupled orthogonal states $H_{\Omega}\left|u_{j}\right\rangle=0$ with $\left\langle u_{j} \mid c\right\rangle=0$ and $\left\langle u_{j} \mid u_{m}\right\rangle$ $=\delta_{j, m}$, for $j, m=1,2, \ldots, N-1$.

For large detuning $\Delta \gg \Omega$ the interaction becomes purely dispersive,

$$
H_{\Omega} \simeq-(\Delta+\delta)|r\rangle\langle r|+\delta| c\rangle\langle c|,
$$

with $\delta=\Omega^{2} / \Delta$. In this case, the desired state is $|c\rangle$. If the system is prepared in the state $|c\rangle$ it will always remain in such a state. Any desired state can be prepared in this way by suitably choosing the intensities and phases of the driving fields. According to the results of the preceding section this preparation will be stable and robust provided that $\delta$ is large enough.

\section{CONCLUSIONS}

We have examined several arrangements for engineering the quantum state of multilevel systems in such a way that the prescribed state is protected against outside perturbations. These arrangements can be regarded as applications of the Zeno and inverse Zeno effects to the preparation and control of quantum systems. The schemes examined are

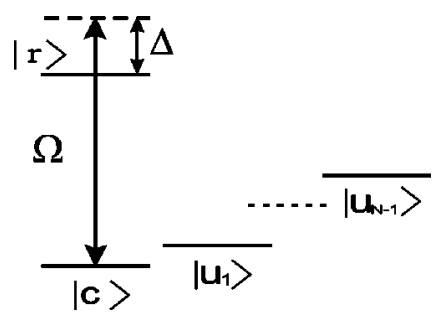

FIG. 6. Level scheme fully equivalent to Fig. 5 in terms of the coupled $(|c\rangle)$ and uncoupled $\left(\left|u_{1}\right\rangle, \ldots,\left|u_{N-1}\right\rangle\right)$ states. 
based on different forms of continuous observation. It is worth stressing that the whole analysis is purely dynamical in terms of joint meter-system evolution equations. We never resort to any of the peculiar features of the measurement in the quantum theory, such as the projection postulate. We have considered schemes based on purely unitary evolution as well as arrangements based on irreversible dynamics. According to our results, all of them are equally suitable for robust coherent trapping.

We point out that these schemes are applicable to real systems. This is because they are based on concepts such as coherent trapping, dark states, and the Zeno effect which have already been tested experimentally in many different atomic systems $[6,8]$. In particular, practical arrangements that can serve to implement the proposed schemes can be found in the context of optical pumping, where the Zeno shielding has already been observed experimentally [13].

As we have mentioned above, these schemes can be of theoretical interest concerning the accurate storage and transfer of information encoded in quantum systems since they avoid alterations of the state of the system. We could include applications to the manipulation of the motional state of trapped ions and their interaction with light fields via the control of the internal state of the ion.
[1] K. Vogel, V. M. Akulin, and W. P. Schleich, Phys. Rev. Lett. 71, 1816 (1993); A. S. Parkins, P. Marte, P. Zoller, and H. J. Kimble, ibid. 71, 3095 (1993); B. M. Garraway, B. Sherman, H. Moya-Cessa, P. L. Knight, and G. Kurizki, Phys. Rev. A 49, 535 (1994); A. S. Parkins, P. Marte, P. Zoller, O. Carnal, and H. J. Kimble, ibid. 51, 1578 (1995); C. K. Law and J. H. Eberly, Phys. Rev. Lett. 76, 1055 (1996); S. Szabo, P. Adam, J. Janszky, and P. Domokos, Phys. Rev. A 53, 2698 (1996); T. Wellens, A. Buchleitner, B. Kümmerer, and H. Maassen, Phys. Rev. Lett. 85, 3361 (2000); R. M. Serra, N. G. de Almeida, C. J. Villas-Bôas, and M. H. Y. Moussa, Phys. Rev. A 62, 043810 (2000).

[2] T. P. Altenmüller and A. Schenzle, Phys. Rev. A 48, 70 (1993); M. Kitano, ibid. 56, 1138 (1997); H. F. Hofmann, G. Mahler, and O. Hess, ibid. 57, 4877 (1998); M. Kitano, K. Yamane, and T. Ikushima, ibid. 59, 3710 (1999).

[3] J. Audretsch, M. B. Mensky, and A. D. Panov, Phys. Lett. A 261, 44 (1999).

[4] D. Home and M. A. B. Whitaker, Ann. Phys. (N.Y.) 258, 237 (1997).

[5] M. Simonius, Phys. Rev. Lett. 40, 980 (1978).

[6] W. M. Itano, D. J. Heinzen, J. J. Bollinger, and D. J. Wineland, Phys. Rev. A 41, 2295 (1990); R. M. Godun, M. B. d'Arcy, M.
K. Oberthaler, G. S. Summy, and K. Burnett, Opt. Commun. 169, 301 (1999).

[7] Y. Aharonov and M. Vardi, Phys. Rev. D 21, 2235 (1980).

[8] E. Arimondo, in Progress in Optics, edited by E. Wolf (NorthHolland, Amsterdam, 1996), Vol. 35, p. 257; J. Marangos, J. Mod. Opt. 45, 471 (1998).

[9] E. Block and P. R. Berman, Phys. Rev. A 44, 1466 (1991); M. J. Gagen and G. J. Milburn, ibid. 47, 1467 (1993).

[10] M. B. Plenio, P. L. Knight, and R. C. Thompson, Opt. Commun. 123, 278 (1996); J. F. Poyatos, J. I. Cirac, and P. Zoller, Phys. Rev. Lett. 77, 4728 (1996); C. J. Myatt, B. E. King, Q. A. Turchette, C. A. Sackett, D. Kielpinsky, W. M. Itano, C. Monroe, and D. J. Wineland, Nature (London) 403, 269 (2000); Q. A. Turchette, C. J. Myatt, B. E. King, C. A. Sackett, D. Kielpinski, W. M. Itano, C. Monroe, and D. J. Wineland, Phys. Rev. A 62, 053807 (2000).

[11] A. Luis and J. Perina, Phys. Rev. Lett. 76, 4340 (1996).

[12] A. Peres, Am. J. Phys. 48, 931 (1980); A. L. Rivera and S. M. Chumakov, J. Mod. Opt. 41, 839 (1994); S. M. Chumakov, K.-E. Hellwig, and A. L. Rivera, Phys. Lett. A 197, 73 (1995); P. Facchi and S. Pascazio, e-print quant-ph/0101044.

[13] S. Slijkhuis, G. Nienhuis, and R. Morgenstern, Phys. Rev. A 33, 3977 (1986); K. Mølhave and M. Drewsen, Phys. Lett. A 268, 45 (2000). 